\title{
Appearance Enhancement Using a Projector-Camera Feedback System
}

\author{
Toshiyuki Amano and Hirokazu Kato \\ Graduate School of Information Science, Nara Institute of Science and Technology \\ \{amano,kato\}@is.naist.jp
}

\begin{abstract}
In this paper, a dynamic appearance enhancement method for the less saturated object is proposed. The appearance enhancement method proposed in this paper is realized with a projector camera dynamic feedback system. Therefore, the proposed method has an ability of the rapid and continuous appearance enhancement and it is useful for the human visual perception assistance. Also, the system not requires a precaptured appearance of the target.
\end{abstract}

\section{Introduction}

The adaptive projection system is realized by the projection without color non-uniformity and shape distortion to the non-uniformity color and non-planar screen. Grossberg [2] proposed a method for controlling an arbitrary object's appearance, and its method made one object look like another. For the geometrical compensation, Bimber [1] proposed general correcting method for geometrical distortion when nonplanar screen is used. Both impressive methods are constructed the base of the adaptive projection technique. Recently, dynamic adaptation became one of the focus of this adaptive projection system [2, 5, 3]. Fujii [2] constructed a coaxial projector camera system whose geometrical distortion is independent for screen shape changes. As the geometric compensation method for user's viewpoint, Tayler [5] proposed real-time modification method of the projection image by the feature tracking of projection image.

The purpose of these methods is to cancel the projection distortion and color non-uniformity for the projection to the non-planar screen that has texture. However, not only compensation but the opposite technique that enhances the non-uniformity is also useful for human visual perception assistance. For example, appearance enhancements of color difference and microscopic defect are useful for visual check of production line. In

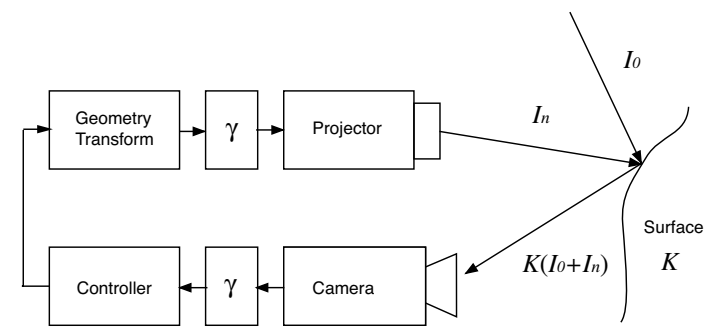

Figure 1. A Pro-Cam Feedback System.

this paper, we describe an innovative technique that enhances an appearance by dynamic feedback system of projector camera system.

\section{Appearance enhancement with projec- tor camera feedback}

The diagram of projector camera feedback system is shown in the figure 1 . The calibration of correspondence between projector and camera coordinate (It is comparable to "Geometry Transform" in the figure.) is a well-known problem, and this correspondence is given by existing method. Therefore, we do not describe about this correspondence problem at the explanation shown in below. As the conceptualistic expression, we can describe a received light energy by camera is in proportion to $\mathcal{K}\left(I_{0}+I_{n}\right)$, when compensation light $I_{n}$ is projected along with the environment light $I_{0}$ on the object's surface of reflection characteristics $\mathcal{K}$. From this relation, the illumination of $(n+1)$ th projection can be expressed by the $n$th projection. In the initial condition, the first compensation light can be written as

$$
I_{1}=\mathcal{P}_{\text {prj }} \Gamma_{\text {prj }} \mathcal{G} \Gamma_{\text {cam }} \mathcal{P}_{\text {cam }} \mathcal{K} I_{0}=\mathcal{G}^{\prime} \mathcal{K} I_{0},
$$

where $\Gamma_{c a m}$ and $\Gamma_{p r j}$ are gamma curve and gamma correction, $\mathcal{P}_{\text {pr } j}$ and $\mathcal{P}_{\text {cam }}$ are converting operators between illumination and pixel value of camera and pro- 
jector respectively. The $\mathcal{G}$ is feedback operator that means feedback gain at the linear model, and $\mathcal{G}^{\prime}$ is feedback operator that includes all camera and projector properties. Since $(n-1)$ th compensation light is projected along with environment light $I_{0}$, the $n$th compensation light is

$$
I_{n}=\mathcal{G}^{\prime} \mathcal{K}\left(I_{0}+I_{n-1}\right)=\sum_{j=1}^{n}\left(\mathcal{G}^{\prime} \mathcal{K}\right)^{j} I_{0}
$$

The final compensation light $\mathbf{I}_{\infty}$ will be

$$
\mathbf{I}_{\infty}=\left(\mathbf{1}-G^{\prime} K\right)^{-1} G^{\prime} K \mathbf{I}_{0}
$$

if we regard a linear feedback system. Where $\mathbf{I}=$ $\left(I_{R}, I_{G}, I_{B}\right)^{T}$ is the illumination power vector of compensation light and the environment light, $G^{\prime}$ is feedback gain matrix, and $K$ is a reflectance matrix that includes reflection characteristics of the surface and chromatic dispersion between RGB channels of projector and camera [4].

\subsection{Brightness and Saturation Control}

From equation 8 , we can see the value of the final compensation light is stable when adequate feedback gain $G^{\prime}$ is given. However, we cannot control appearance brightness, saturation, and contrast independently. For this reason, we use fractional input such as

$$
I m_{*}^{\prime}=\frac{3 I m_{*}}{I m_{R}+I m_{G}+I m_{B}},
$$

as the input image for the feedback system to make it cancel the contrast in the $I m_{*}$. Where $I m_{*}$ is brightness level of captured image, $* \in\{R, G, B\}$ is index of color channel. With this fractional input, we use

$$
P_{*}(x, y)=3 g\left(k \operatorname{Im}_{*}^{\prime}+\frac{1}{3}(1-k)\right)
$$

as a feedback controller, to control the object's brightness and saturation. The parameter $k$ controls the saturation of the object's appearance and that means blending ratio of white light and chromatic image. The parameter $g$ controls the brightness of the enhanced object's appearance.

\subsection{Contrast Control}

We implemented the brightness and saturation control of the object's appearance by using of fractional feedback. However, not only brightness and saturation but also the contrast control is effective for appearance enhancement. Therefore, we add the contrast control by the modification of the feedback gain $g$ for each pixel $(x, y)$ according to the brightness deviation in $\mathrm{Im}_{*}$ such as

$$
g^{\prime}(x, y)=g+g_{c}(\overline{\operatorname{Im}}(x, y)-c),
$$

where $\operatorname{Im}(x, y)$ is brightness of each pixel, ( That is $\left.\left(I m_{R}+I m_{G}+I m_{B}\right) / 3\right), c$ is average brightness of the image $\operatorname{Im}(x, y)$. The parameter $g_{c}$ is the parameter of the contrast enhancement.

\subsection{Convergence Value Analysis}

The fractional feedback system is described in the linear matrix by using of the homogeneous expression $\mathbf{I}^{\prime}=\left(I_{R}, I_{G}, I_{B}, 1\right)^{T}$, and the output of projector is

$$
\mathbf{I}_{n+1}^{\prime} \sim\left[\begin{array}{cccc}
g & 0 & 0 & 0 \\
0 & g & 0 & 0 \\
0 & 0 & g & 0 \\
1 & 1 & 1 & 0
\end{array}\right]\left[\begin{array}{cc}
K & \mathbf{0} \\
\mathbf{0} & 1
\end{array}\right] \mathbf{I}_{n}^{\prime}
$$

If we omit chromatic dispersion, the final compensation light is written as

$$
\begin{aligned}
\mathbf{I}_{\infty}^{\prime} & =\left(1-G^{\prime} K\right)^{-1} G^{\prime} K \mathbf{I}_{0}^{\prime} \\
& =\left[\begin{array}{cccc}
\frac{g k_{R}}{1-g k_{R}} & 0 & 0 & 0 \\
0 & \frac{g k_{G}}{1-g k_{G}} & 0 & 0 \\
0 & 0 & \frac{g k_{B}}{1-g k_{B}} & 0 \\
\frac{k_{R}}{1-g k_{R}} & \frac{k_{G}}{1-g k_{G}} & \frac{k_{B}}{1-g k_{B}} & 0
\end{array}\right] \mathbf{I}_{0}^{\prime},
\end{aligned}
$$

where $K=\operatorname{diag}\left(k_{R}, k_{G}, k_{B}\right)$. From eq.(8), we can confirm that the brightness of final compensation light is converging according to the feedback gain and the fractional feedback system is stable.

\section{Simulation and Results}

\subsection{Step Response Simulation}

If the object whose appearance is enhanced moved quickly, the color at one pixel changes immediately. Thus, we checked the step response of the proposed feedback system by the numeric simulation to evaluate system tracking ability. The figure 2 shows step response. In the figure $2, I_{R 0}, I_{G 0}$ and $I_{B 0}$ are normalized illumination under the $I_{0}$ (these are comparable to the reflectance ratio on the object surface.), $I_{R}, I_{G}$ and $I_{B}$ are observed light power of each channel, horizontal axes are compensation steps that is meaning acquired frame number. These simulations were started with an initial condition (without the compensation light), and the color of the object changes quickly in the step number 6 . The feedback gain $g=0.5$, camera gamma value 


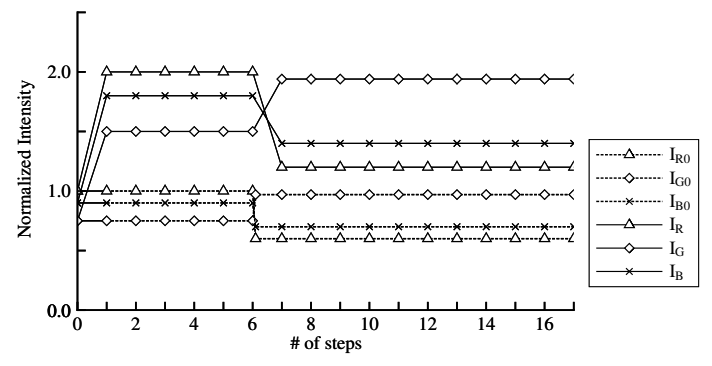

(a) In case of $g=0.5, k=0$

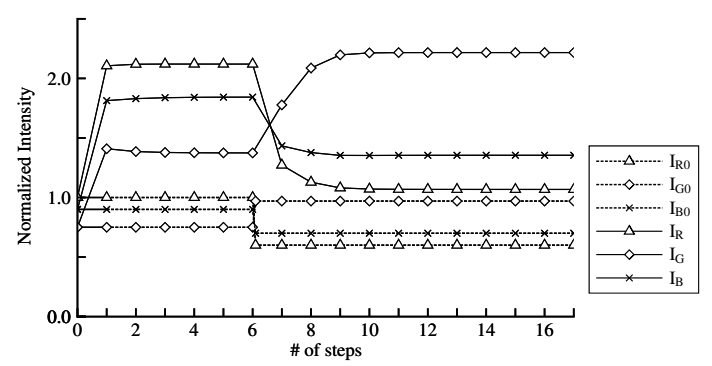

(b) In case of $g=0.5, k=0.8$

Figure 2. Step Response of the System.

$\gamma=1.0$ and we regard the illumination response curve of the projector as linear.

The figure 2 shows the step responses of $k=0$ and $k=0.8$. From these results, we can see that observed illuminations are following object illumination under the $\mathbf{I}_{0}$, and the disparity of RGB channels is increasing along with the parameter $k$ and that means the saturation is increasing. The responses of $I_{R}, I_{G}$ and $I_{B}$ are going stable in 3 to 4 steps after the step input at the step number 6 . From simulations by the various gamma value 0.1 to 1.0 , the response by the $\gamma=1.0$ was the slowest. Thus, we can estimate the system response as about $0.4 \mathrm{sec}$ in the $10 \mathrm{fps}$.

\subsection{Experimental Results}

Figure 3 shows the experimental setup. A camera (SONY XCD-710CR, RGB 8bit, $1024 \times 768$ resolution, $\gamma=1$ ) is attached with the projector (CANON LV7365, RGB 8bit, $1024 \times 768$ resolution) and the compensation light is projected at a slant from above under the fluorescent light. If we use the coaxial projector camera configuration, the correspondence of projector pixels and camera pixels becomes independent with object's shape, and the optical compensation for moving 3-D object is possible. However, in this research

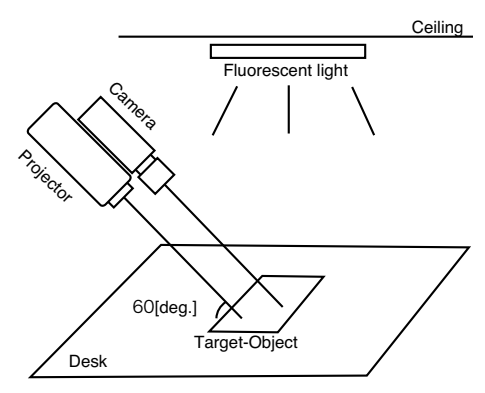

Figure 3. System setup.

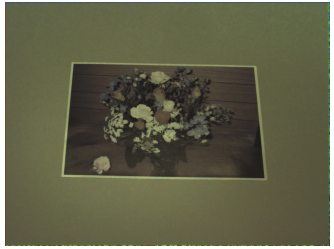

(a) Captured $I m_{0}$

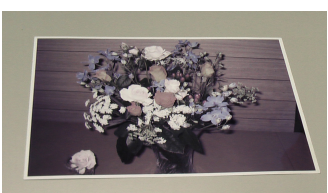

(c) Original Appearance

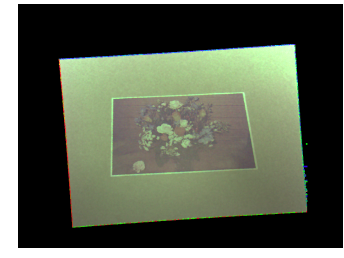

(b) Compensation Pattern

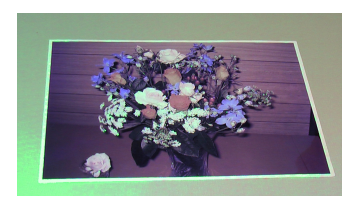

(d) Enhanced Appearance
Figure 4. Enhancement for Faded Picture.

we use non-coaxial optics and execute the dynamical appearance enhancement for the planar object, because we focus on the evaluation of appearance enhancement in this experiment.

At the first, we calibrated the pixel correspondence between acquired image and projection image by the projection of gray code images. Next, we calibrated the color space between the camera and the projector by the linear RGB-RGB mapping by the averaged pixels value. And after, we calibrated feedback gain and lens vignetting by the image that captured with white light projection.

The figure 4 shows the enhancement results for faded pictures. The figure 4(a) is the captured image under the environment light without compensation pattern projection, and the figure 4(b) is the compensation pattern that enhances object's saturation $\left(g=0.25, k=0.4, g_{c}=\right.$ 0 ). The figure 4(c) is the original appearance under the environment light and we got the appearance enhancement result with the compensation pattern projection that is shown in the figure 4(d). These enhancements were executed sequentially at a speed of $15 \mathrm{fps}$ by the Quad Core 2.66GHz PC (Linux), and the compensation 


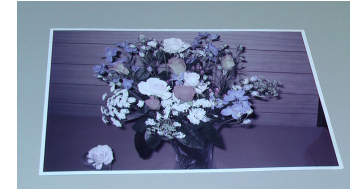

(a) $k=0$

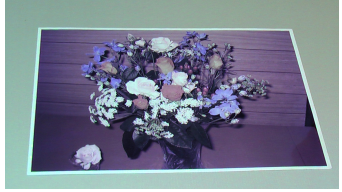

(c) $k=0.30$

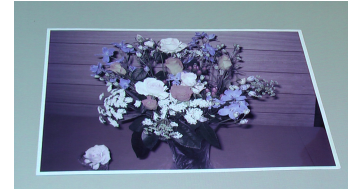

(b) $k=0.15$

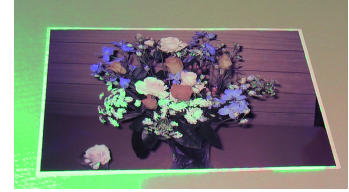

(d) $k=0.45$
Figure 5. Saturation Enhancement.

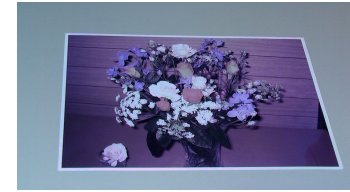

(a) $g_{c}=0$

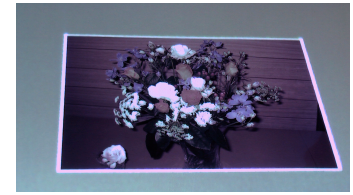

(c) $g_{c}=0.5$

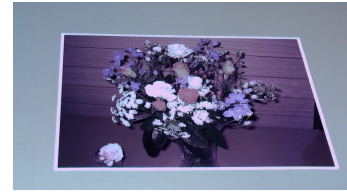

(b) $g_{c}=0.3$

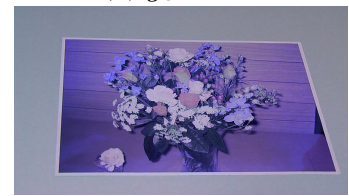

(d) $g_{c}=-0.06$
Figure 6. Contrast Enhancement.

image pattern was stable within 5 steps for static scene. Therefore, the response time by our experimental equipment was $0.3 \mathrm{sec}$.

The figure 5 shows the saturation enhancement results $\left(g=0.25, g_{c}=0\right)$. The saturation of the faded color picture was enhanced by our system. We can see its appearance become vivid along with the increase of the parameter $k$. The figure 6 shows the enhanced results of the contrast by various parameters $(g=$ $0.25, k=0.40)$. The parameter $g_{c}$ controls the contrast of the object's appearance. The contrast is increasing with the increase of the $g_{c}$, and the appearance of solidity is increasing because the shadow and highlight are enhanced. And also, the appearance is changing to matte with decrease with the $g_{c}$.

\section{Discussion}

The proposed method is efficient because it is able to control the object appearance in the real world and sequentially. In our life, we are using our visual perception always. Thus, We think the appearance enhance- ment is required by many applications, not only visual check assistance for production line, for example, perception assistance for color amblyopia, costume design assists for apparel industry, color rendering control for stage lighting and other many applications. The proposed method gives the control of the object appearance of the brightness, saturation, and contrast independently, and that is matching with human perception sense. Therefore, it is useful for many applications, but the color compensation non-uniformity that caused lens aberration in the figure 5(b). And for the optical configuration, the enhancement restricts a possible object only to the plane object. We would like to solve these problems in a future work.

\section{Conclusion}

In this paper, we proposed an appearance enhancement method by the projector camera feedback system. This enabled the enhancements of object's appearance of brightness, saturation, and contrast in sequentially at the real world. The remaining problems are the correction of non-uniformity of brightness and color calibration, and we would like to adopt co-axial optics for appearance enhancement for the non-plane objects in the future work.

\section{References}

[1] O. Bimber, G. Wetzstein, A. Emmerling, C. Nitschke, and A. Grundhöfer. Enabling view-dependent stereoscopic projection in real environments. In SIGGRAPH '05: ACM SIGGRAPH 2005 Sketches, page 120, New York, NY, USA, 2005. ACM.

[2] M. D. Grossberg, H. Peri, S. K. Nayar, and P. N. Belhumeur. Making one object look like another: Controlling appearance using a projector-camera system. cvpr, 01:452-459, 2004.

[3] A. Grundhöfer, M. Seeger, F. Häntsch, and O. Bimber. Dynamic adaptation of projected imperceptible codes. In IEEE International Symposium on Mixed and Augmented Reality (ISMAR'07), pages 181-190, Washington, DC, USA, 2007. IEEE Computer Society.

[4] A. Ilie and G. Welch. Ensuring color consistency across multiple cameras. In ICCV '05: Proceedings of the Tenth IEEE International Conference on Computer Vision, pages 1268-1275, Washington, DC, USA, 2005. IEEE Computer Society.

[5] T. Johnson and H. Fuchs. Real-time projector tracking on complex geometry using ordinary imagery. In $C V P R W$ '07: Proceedings of the 2007 Conference on Computer Vision and Pattern Recognition Workshop, page 6, Washington, DC, USA, 2007. IEEE Computer Society. 